\title{
Análisis teórico y empírico acerca de la dirección de comunicación en la empresa
}

\author{
María Concepción PARRA-Meroño \\ UCAM-Universidad Católica San Antonio de Murcia \\ mcparra@ucam.edu \\ Beatriz PEÑA-ACUÑA \\ UCAM-Universidad Católica San Antonio de Murcia \\ bpena@ucam.edu \\ Gonzalo WANDOSELL-FERNÁNDEZ-DE-BOBADILLA \\ UCAM-Universidad Católica San Antonio de Murcia \\ gwandosell@ucam.edu
}

\begin{abstract}
Resumen:
Esta investigación pretende adentrarse en la cultura empresarial española a través de una investigación llevada a cabo en una muestra de 40 alumnos con experiencia profesional que han terminado de cursar recientemente la asignatura de "Dirección de comunicación y habilidades directivas" en el curso 20122013. En este trabajo se les pregunta sobre la dirección de comunicación en la empresa. El objetivo es conocer el estado en el que se encuentra una representación de la cultura empresarial española a partir de la experiencia personal de los encuestados, tanto en su etapa inicial, mientras desarrollaban sus estudios de grado como en personas con experiencia profesional. Los resultados indican que en la mayoría de las empresas investigadas no existe la figura del Director de Comunicación, aunque sí hay alguna persona encargada de realizar su función.
\end{abstract}

Palabras clave: Dirección de comunicación, cultura empresarial, comunicación interna, comunicación corporativa, y nuevas tecnologías.

\section{Theoretical and empirical analysis about the direction of corporate communication}

\begin{abstract}
This research aims to penetrate the Spanish business culture through research carried out in a sample of 40 students with professional experience who have recently terminated at the subject "E-communication and managerial skills" during 2013-2013. In this paper we will address questions about communication within the company. The research aim is to know the state in which there is a representation of the Spanish business culture from the personal experience of the respondents, both in their initial stage, while developing his degree as well with professional experience. The results indicate that in most of the Spanish investigated companies there is still no figure of the Director of Communication, although there is a person in charge of performing this function.
\end{abstract}

Key Words: Communication management, corporate culture, internal communication, corporate communication and New Technologies. 


\section{Referencia normalizada:}

Parra Meroño, M. C.; Peña Acuña, B.; Wandosell Fernández de Bobadilla, G. (2013) Análisis teórico y empírico acerca de la dirección de comunicación en la empresa. Historia y Comunicación Social. Vol. $18 \mathrm{~N}^{\circ}$ Especial Octubre. Págs. 445-453.

Sumario: 1. Introducción. 2. La dirección de comunicación. 3. Metodología. 4. Resultados de la investigación. 5. Conclusiones. 6. Bibliografía.

\section{Introducción}

Este trabajo pretende realizar un acercamiento a la cultura empresarial española a través de una investigación llevada a cabo en una muestra de alumnos con experiencia profesional -que alternan junto con los estudios universitarios- que han terminado de cursar recientemente la asignatura de "Dirección de comunicación y habilidades directivas". Buscamos indagar acerca de la figura del Director de Comunicación en la empresa, de los valores que transmite la empresa a través de su imagen corporativa y de los medios de comunicación más utilizados para la comunicación interpersonal, a tres niveles, descendente, horizontal y ascendente. Finalmente, también se pregunta sobre la coherencia entre la imagen interna y externa de la empresa.

La muestra se compone de 40 alumnos de Cuarto curso de Grado de Administración y Dirección de Empresas. La metodología es cuantitativa y cualitativa, pues se les interrogó a través de un cuestionario semi-estructurado (que se puede consultar en el Anexo).

\section{La dirección de comunicación}

La sociedad actual ha pasado a denominarse Sociedad de la Comunicación, debido al auge de los medios de comunicación y sobre todo, de los nuevos medios auspiciados por el uso generalizado de internet. Ahora nos comunicamos a través de las redes sociales, como Facebook, leemos la prensa en internet, nos escribimos por correo electrónico, etc. En definitiva, la comunicación está cada vez más presente en nuestra vida y somos más conscientes de su necesidad. Se concibe así la Sociedad de la Comunicación como una prioridad en la actividad social y productiva.

Sin embargo, en el ámbito de la empresa, aún queda mucho por hacer, de modo que los directivos adquieran esa mentalidad comunicativa que otorgue al Director de Comunicación (DirCom) el papel preponderante que debería ocupar, dada la importancia que a nivel societario ha adquirido la Comunicación. De hecho, una empresa puede tener el mejor producto y aun así, no tener éxito. Sin duda, eso se debe a que no es capaz de comunicar a su público objetivo las bondades y necesidades que se pueden alcanzar con la compra y uso de dicho producto (Parra y Beltrán, 2011). 
La comunicación consiste en la interacción entre personas para transmitir o intercambiar información, ideas o sentimientos. Esta interacción es el fundamento de toda vida social, hasta el extremo de que si se suprime en un grupo social todo intercambio de signos orales o escritos, el grupo desaparece.

Entendemos la comunicación empresarial como el intercambio planificado de mensajes dentro y fuera de la empresa. Este intercambio permite proyectar la imagen y el funcionamiento de la empresa hacia el exterior, así como conocer a los individuos que trabajan en ella, lo que se hace para que sus miembros participen y se sientan implicados con la empresa y con sus valores (Arellano, 2008:1-2).

Es tarea del Director de Comunicación ejecutar el plan estratégico de comunicación de la empresa y lograr que sea eficaz, que no quede en un documento sin más. Así, este directivo, al influir sobre los demás, podrá propiciar el éxito de ese plan y por ende, de la empresa. El DirCom debe también cerciorase de que los flujos de comunicación de la empresa fluyan y se realicen de acuerdo a unos planes prefijados. Además, es responsable de la imagen corporativa de la empresa, fuera y dentro de la compañía, de lograr la coherencia entre la imagen externa que se proyecta y la que se vive en el interior de la organización, con el objetivo de que todos los miembros de la organización sean comunicadores de los valores y la visión de la empresa.

Además, recordamos que los "cuatro tipos de habilidades deseables en un Dircom: comunicativas, directivas, emocionales, interpersonales y dominio del lenguaje no verbal. El DirCom debe desarrollar habilidades directivas y sociales, para realizar un trabajo de relaciones públicas con los contactos internos y externos; de liderazgo, para crear una buena sinergia de implicación entre los empleados y que acojan la cultura empresarial; celo y habilidades para efectuar un buen marketing, consolidar la imagen corporativa y los valores de la empresa. Asimismo, es conveniente que sea hábil para reconocer el lenguaje no verbal, así como el lenguaje paralingüístico de los demás" (Peña, 2005: 2).

Un trabajo previo a esta investigación, El estado de la Comunicación en España 2010, realizado por la Asociación de Directivos de Comunicación, resulta interesante pues permite conocer al DirCom español, su estatus y las competencias que ostenta, la importancia que percibe de la comunicación empresarial, la presencia de su papel en las grandes compañías, si se planifica mediante el Plan de Comunicación, la estructura y funciones del Departamento de comunicación, en qué se forman e investigan los DirCom y qué margen le dan a la Consultoría externa. También existe otra investigación europea llamada European Communication monitor 2009, en la que se realiza un estudio más extenso acerca de la profesión de comunicación y las relaciones públicas, que recoge las opiniones de 2.000 profesionales de 34 países.

Según Manuel Gross, la cultura organizacional consiste en "el conjunto de percepciones, sentimientos, actitudes, hábitos, creencias, valores, tradiciones y formas de interacción dentro y entre los grupos existentes en todas las organizaciones. En este conjunto de conceptos están representadas las normas informales y no escritas que orientan el comportamiento cotidiano de los miembros de la orga- 
nización, comportamientos que pueden o no estar alineados con los objetivos de la organización" (Gross, 2009: 1).

\section{Metodología}

El estudio empírico realizado se ha llevado a cabo administrando un cuestionario semi estructurado a una muestra de 40 alumnos que han cursado recientemente la asignatura de Comunicación y habilidades directivas de cuarto curso de Grado en Administración y Dirección de empresas. Dicho cuestionario, permite adoptar dos enfoques de investigación, uno cuantitativo, mediante preguntas cerradas y otro cualitativo, mediante cuestiones abiertas, las cuales permiten una mayor flexibilidad así como una mayor riqueza de la información recopilada.

El cuestionario está compuesto de tres apartados. El primero hace referencia a los datos de clasificación de los encuestados (edad, sexo y modalidad de estudios); así como a los datos de las empresas sobre las que se realiza la encuesta (tamaño, ubicación y actividad empresarial que desarrollan). El segundo alude a la Dirección de comunicación en la empresa y está compuesto por preguntas relativas al departamento de comunicación, la imagen corporativa, los valores empresariales identificables a través de la imagen y los medios de comunicación disponibles para la comunicación descendente, horizontal y ascendente. La tercera parte se centra en el liderazgo directivo. En ella, se identifican los diferentes estilos de liderazgo ejercidos por los directivos empresariales y los mandos intermedios. Esta parte no será objeto de estudio en este trabajo.

Las personas entrevistadas son 40 estudiantes con experiencia laboral en empresas de diferentes sectores de actividad, siendo un 50\% hombres y un 50\% mujeres, con una edad media de 29,25 años. De ellos, el 52,5\% han realizado el Curso de Adaptación al Grado en Administración y Dirección de Empresas, mientras que el resto $(47,5 \%)$ son estudiantes del Grado de ADE. De ahí, la media de edad de los encuestados.

Las empresas investigadas, aquellas en las que los estudiantes han ejercido su actividad laboral, son en su gran mayoría del sector servicios (asesoría, banca, atención al cliente, comercio, etc.), pero también de la industria y de la agricultura. Por lo que se refiere al tamaño de la empresa, la mayoría son pequeñas empresas, de menos de 50 empleados $(59 \%)$.

\section{Resultados de la investigación}

En este epígrafe se detallan los resultados del estudio empírico relativos a la Dirección de la Comunicación en la empresa. A continuación, la Tabla 1 muestra los resultados de la función de comunicación en la empresa. 
Tabla 1. La dirección de comunicación en la empresa

\begin{tabular}{|l|l|l|}
\hline La función de comunicación en la empresa & No & Sí \\
\hline ¿Hay un departamento de comunicación? & 79,5 & 20,5 \\
\hline ¿Hay un director de comunicación? & 76,3 & 23,7 \\
\hline ¿Alguien en la empresa lleva la comunicación? & 31,2 & 68,8 \\
\hline ¿Se fomenta la cultura de comunicación? & 35,9 & 64,1 \\
\hline ¿Se cuida la imagen corporativa? & 25,0 & 75,0 \\
\hline
\end{tabular}

Los resultados expuestos en la Tabla 1 muestran que en la mayoría de las empresas no existe un departamento de comunicación como tal en el 79,5\% de los casos, ni tampoco un director de comunicación, en el 76,3\% de los casos. Sin embargo, sí que existe alguien que se encarga de la comunicación de la empresa, en un $68,8 \%$ de las empresas. Además, en un $64,1 \%$ de ellas se fomenta la cultura de comunicación y se cuida la imagen corporativa en un $75 \%$ de las empresas investigadas. La persona encargada de la comunicación en la empresa se ubica preferentemente en Dirección (47,9\%), seguido por el departamento de Marketing (26,1\%), Comunicación y Publicidad (13\%) y en Recursos Humanos (13\%). Los resultados son coherentes con la cuestión anterior, dado que hay muchas empresas en las que no existe la figura del Director de Comunicación como tal.

Además, se pedía que indicaran tres valores de la empresa a través de su imagen. En este apartado las respuestas han sido muy variadas, pero hay cuatro tipos de valores claramente identificados con un reparto prácticamente similar entre los 120 señalados por los entrevistados: los centrados en el cliente (servicio al cliente, atención personalizada, compromiso, confidencialidad, exclusividad), los centrados en los visión de la empresa y sus recursos humanos (seriedad, compromiso, compañerismo, profesionalidad, responsabilidad, etc.), los centrados en el producto (calidad, innovación, envase, precio, diferenciación) y finalmente, aquellos enfocados en los beneficios empresariales (trabajo, competitividad, solvencia, valor añadido, etc.).

La siguiente cuestión analizada son los medios de comunicación empleados para la comunicación descendente, horizontal y ascendente.

Para la comunicación descendente la Tabla 2 muestra los tipos de comunicación disponibles.

Tabla 2. Medios de comunicación descendente

\begin{tabular}{|l|c|c|}
\hline & Frec. & $\mathbf{\%}$ \\
\hline Correo electrónico & 29 & 35,0 \\
\hline Entrevistas personales & 24 & 28,9 \\
\hline Reuniones periódicas & 24 & 28,9 \\
\hline Otros (Intranet, teléfono) & 6 & 7,2 \\
\hline Total & 83 & 100 \\
\hline
\end{tabular}


Como puede observarse (Tabla 2), el medio de comunicación descendente más empleado es el correo electrónico, seguido muy de cerca por las entrevistas personales y las reuniones periódicas.

Por lo que se refiere a la comunicación horizontal (Tabla 3) los medios de comunicación más utilizados son por este orden, las reuniones periódicas y el correo electrónico. Le siguen a gran distancia los famosos tablones de anuncios y otros medios (teléfono, carta, etc.).

Tabla 3. Medios de comunicación horizontal

\begin{tabular}{|l|c|c|}
\hline & Frec. & \% \\
\hline Correo electrónico & 25 & 35,2 \\
\hline Reuniones periódicas & 31 & 43,7 \\
\hline Tablón de anuncios & 8 & 11,3 \\
\hline Otros (Intranet, teléfono) & 7 & 9,8 \\
\hline Total & 71 & 100 \\
\hline
\end{tabular}

Finalmente, en cuanto a la comunicación ascendente, la Tabla 4 muestra los medios utilizados en las empresas analizadas. Se puede observar la preponderancia de los medios tradicionales en este tipo de comunicación, la entrevista personal y las reuniones periódicas. A mucha distancia se encuentran tanto el buzón de sugerencias como la encuesta de satisfacción laboral.

Tabla 4. Medios de comunicación ascendente

\begin{tabular}{|l|c|c|}
\hline & Frec. & \% \\
\hline Entrevista personal & 27 & 36 \\
\hline Buzón de sugerencias & 9 & 12 \\
\hline Encuesta anónima de satisfacción laboral & 8 & 10,7 \\
\hline Reuniones periódicas & 26 & 34,7 \\
\hline Otros & 5 & 6,6 \\
\hline Total & 75 & 100 \\
\hline
\end{tabular}

Sorprende, que tan solo se hayan identificado ocho casos de encuesta de satisfacción laboral, lo que podría indicar que no hay un gran interés por parte de los directivos y mandos intermedios en conocer la opinión de los trabajadores, puesto que tampoco se suele utilizar el buzón de sugerencias.

Finalmente, en la mayoría de los casos (75\%), se percibe que la imagen corporativa que se trata de dar al exterior es coherente con la que hay dentro de la empresa, tanto por lo que ocurre en la cultura de la empresa como por lo que se refleja a través de la comunicación interna. 


\section{Conclusiones}

Nuestros resultados indican que en la mayoría de las empresas investigadas no existe un departamento de comunicación como tal, en el 79,5\% de los casos, al igual que no un director de comunicación. Sin embargo, sí hay un encargado de la comunicación, que suele depender de la Dirección de la empresa. Estos datos indican, que cada vez se le da más importancia al área de comunicación en la empresa, puesto que suele haber un encargado de la tarea de comunicación y además, los directivos prefieren supervisar directamente esta tarea, que encomendarla a otros departamentos.

Por otra parte, también hemos hallado evidencia de que los valores que transmiten las empresas a partir de su imagen corporativa son muy variados, y que se pueden agrupar en cuatro categorías diferenciadas, los centrados en el cliente (servicio al cliente, atención personalizada, compromiso, confidencialidad, exclusividad), los centrados en los visión de la empresa y sus recursos humanos (seriedad, compromiso, compañerismo, profesionalidad, responsabilidad, etc.), los centrados en el producto (calidad, innovación, envase, precio, diferenciación) y finalmente, aquellos enfocados en los beneficios empresariales (trabajo, competitividad, solvencia, valor añadido, etc.).

Los medios de comunicación más utilizados por las empresas varían en función del tipo de comunicación de que se trate, ya sea descendente, horizontal o ascendente.

Así, para la comunicación descendente el más empleado es el correo electrónico, seguido muy de cerca por las entrevistas personales y las reuniones periódicas, lo que es un signo claro de la presencia de las nuevas tecnologías en los sistemas de comunicación interpersonal en el ámbito empresarial. Por lo que se refiere a la comunicación horizontal el medio de comunicación más empleado es la reunión periódica, seguida por el correo electrónico, siendo mucho menos habitual el tradicional tablón de anuncios. En lo que se refiere a la comunicación ascendente, lo más utilizado son las reuniones periódicas, seguidas muy de cerca por la entrevista personal. No obstante, al menos en las empresas españolas investigadas no se suele emplear muy a menudo la encuesta de satisfacción laboral ni tampoco el buzón de sugerencias. Esto puede ser un síntoma de que no hay un gran interés por conocer la opinión de los empleados, lo que sin duda es un error, puesto que un buen clima laboral, en el que se fomente la comunicación en todos los sentidos, tanto horizontal, como ascendente repercutirá favorablemente en los logros de la empresa y hará que todos los miembros de la organización se sientan identificados con los valores y la visión de la organización.

Finalmente, debemos destacar que nuestros hallazgos no pueden generalizarse a todas las organizaciones empresariales, puesto que el tamaño muestral es pequeño, pero si, a través de este pequeño ejemplo, se puede dar una idea de la cultura de la comunicación que existe en España. 


\section{Referencias bibliográficas}

PARRA MEROÑO, M. C. y BELTRÁN BUENO, M.A. (2011). Marketing y Dirección Comercial. Murcia: Universidad Católica San Antonio.

Artículos en publicaciones web

ARELLANO, E. (2008). "La estrategia de comunicación orientada al desarrollo de la cultura organizacional". En: Revista Razón y Palabra, n $\mathrm{n}^{\circ}$ 62. pp.1-15. Disponible en http://www.razonypalabra.org.mx/n62/varia/earellano.html. [12-07-2013].

ASOCIACIÓN DE DIRECTIVOS DE COMUNICACIÓN (2010). El estado de la Comunicación en España 2010. Disponible en http://www.dircom.org/publicaciones/estudios. [12-07-2013].

GROSS, M. (2009). Definición y características de la cultura organizacional. Disponible en http://manuelgross.bligoo.com/definicion-y-caracteristicas-de-la-cultura-organizacional-actualizado. [12-07-2013].

PEÑA ACUÑA, B. (2005). "La dirección de comunicación y las habilidades directivas". En: Revista de la Comunicación de la SEECI, n 12, año VII. Páginas 36-54. http://www.seeci.net/Numeros/Numero\%2012/DATOS.html\#ladi. [12-07-2013].

\section{Anexo}

\section{ENTREVISTA SEMIESTRUCTURADA}

Contesta a las preguntas con una cruz X y con sinceridad acerca de tu visión sobre de la Dirección de Comunicación y el Estilo de Liderazgo de una empresa real que conozcas de cerca.

ESTA ENTREVISTA SE UTILIZARÁ CON FINES DE INVESTIGACIÓN.

MUCHAS GRACIAS POR TU COLABORACIÓN.

Tus datos serán tratados de forma anónima de acuerdo con la Ley 15/1999 de Protección de Datos de Carácter Personal.

¿Varón o mujer?

Edad

ESTUDIOS PREVIOS

NÚMERO APROXIMADO DE EMPLEADOS EN LA EMPRESA

LUGAR DE LA EMPRESA REGIÓN

SECTOR AL QUE PERTENECE (ASESORIA, TRANSPORTE, BANCA, ETC.)

\section{PRIMERA PARTE: ACERCA DE LADIRECCIÓN DE COMUNICACIÓN EN LAEMPRESA}

1.¿Hay departamento de comunicación en la empresa?

a. si

b. no

2.¿Hay un director de comunicación en la empresa?

a. si

b. no 
3.En caso afirmativo, ¿de quién depende jerárquicamente directamente?

4.En caso afirmativo, ¿qué formación tiene?

a. Periodista

b.Publicista

c.Otros

5.En caso de que no ¿alguien de la empresa lleva la comunicación?

a. si

b. no

6.En caso de que sí ¿en qué departamento está?

7. ¿Consideras que en esa empresa se fomenta la cultura de la comunicación entre los que la constituyen?

8. ¿Consideras que se cuida la imagen corporativa?

9.¿Puedes enumerar tres valores de esta empresa a partir de su imagen?

10.¿De qué medios se dispone para la comunicación descendente? (PUEDE MARCAR MÁS DE 1)

a.Correo electrónico

b.Entrevistas personales

c.Reuniones periódicas

d.Otros: Especifica, por favor

11. ¿De qué medios se dispone para la comunicación horizontal? (PUEDE MARCAR MÁS DE 1)

a.Correo electrónico

b.Reuniones periódicas

c. Tablón de anuncios

d.Otros. Especifica, por favor,

12.¿De qué medios se dispone para la comunicación ascendente? (PUEDE MARCAR MÁS DE 1)

a.Entrevista personal

b.Buzón de sugerencias

c. Encuesta anónima de satisfacción del trabajador

d.Reuniones periódicas

e.Otros. Especifica, por favor,

13. ¿Es coherente la imagen que se trata de dar al exterior (comunicación externa) con la que hay dentro de la empresa (por lo que ocurre en la cultura de la empresa y por lo que se refleja a través de la comunicación interna)?

\section{SEGUNDA PARTE: ACERCA DEL ESTILO DE LIDERAZGO EN LA EMPRESA.}

14.¿Qué estilo de liderazgo hay entre los directivos de la empresa? (PUEDE MARCAR MÁS DE 1)

a.Liderazgo dictador

b.Liderazgo autocrático

c.Liderazgo democrático

d.Liderazgo paternalista 